\title{
Diagnosis of Knee Joint Osteoarthritis by Bioelectrical Impedance Plethysmography
}

\author{
Rajendra Kumar Saxena ${ }^{1}$ Sneh Anand ${ }^{1}$ Sujoy Kumar Guha ${ }^{1}$ \\ ${ }^{1}$ Centre for Biomedical Engineering, Indian Institute of Technology, \\ New Delhi, India \\ J Health Allied Sci ${ }^{N U}$ 2022;12:376-384. \\ Address for correspondence Rajendra Kumar Saxena, PhD, Centre for \\ Biomedical Engineering Indian Institute of Technology, New Delhi \\ 110016, India (e-mail: rksaxena1943@gmail.com).
}

\author{
Abstract \\ Keywords \\ - knee joint \\ - diagnosis \\ - electrical impedance \\ plethysmography \\ - osteoarthritis
}

Diagnosis of knee joint osteoarthritis $(\mathrm{KJO})$ at early stages can prolong the progression of disabling degenerative ailments. Mostly, diagnosis of $\mathrm{KJO}$ is based on patient complaints about difficulty with locomotion and is clinically diagnosed based on gait and features. Classical radiographic and MRI images further validated the presence of KJO. Due to the economic and limited accessibility of the above services in India and neighboring countries, a large number of patients with $\mathrm{KJO}$ continue to suffer without a diagnosis. There is a need to develop a cost-effective, quick, simple, non-invasive, and reliable diagnostic technique for the onset of $\mathrm{KJO}$. The present study aims to monitor the conductivity changes and impedance fluctuations using an electrical impedance plethysmograph. Impedance fluctuations recorded in normal and osteoarthritis patients showed marked differences. The analysis of these records can identify early damage to the joint.

\section{Introduction}

Osteoarthritis is a common pathological condition affecting knee joints. In Asia, especially in India, knee joint osteoarthritis $(\mathrm{OA})$ is a common disability of weight-bearing joints due to socio-religious reasons and way of life. People generally sit on the floor in a squatting posture for most of the time for routine work and when using the toilet. In Western countries, hip joint osteoarthritis is a common cause of disability. ${ }^{1,2}$ Obesity, trauma, genetic factors, race, etc., have been suggested as risk factors for the genesis of $\mathrm{OA}^{3,4}$ It generally affects the elderly population and is more common and severe in women than in men. Several reasons, such as anatomic differences in hip structures, previous trauma, and genetic and hormonal issues have been proposed for female knee joint agony. The anatomic differences between males and females that may play a role include narrower femurs, thinner patellae, larger quadriceps angles, and differences in tibial condylar size. ${ }^{5}$ Also, the effect of menopause, i.e., paucity of estrogen may be a contributing factor. $^{6}$

Knee joint articular cartilage is an avascular, aneuronic, and metabolically active tissue composed of extracellular matrix (collagen and proteoglycans) and sparsely distributed cells. ${ }^{7}$ In normal articular cartilage, tissue fluid represents 65 to $80 \%$ of the total weight. ${ }^{8}$ Inorganic ions such as sodium, calcium, chloride, and potassium are also found in the fluid phase and have a fixed charge density. ${ }^{9}$ Articular cartilage also exhibits creep and stress-relaxation responses during motion. ${ }^{10}$ During the normal articulation of the knee, joint loading displaces the ionic synovial fluid to the sides, causing changes in the electrical conductivity. ${ }^{11}$ Degeneration of avascular hyaline porous cartilage is due to various etiological factors (aging, mechanical stresses, etc.) that generate lesions in the articular matrix, that is, pits, crevices, and micro cleft formation. ${ }^{12,13}$ During the process of published online February 3, 2022
DOI https://doi.org/ $10.1055 / \mathrm{s}-0041-1741562$ ISSN 2582-4287.

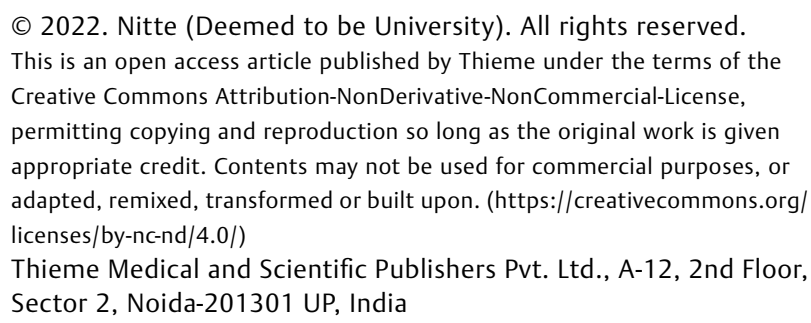


degradation, several proteolytic enzymes and kinins are released by chondrocytes, altering the viscous and biochemical properties of synovial fluid. ${ }^{14}$ Inflammatory processes further enhance the degradation process of the cartilage matrix and the severity of $\mathrm{OA}$.

Early detection of KJO may help in the initiation of early interventions and therapeutic methodologies, which may control the progression and severity of the disease. Latestage diagnosis of OA when occurs when the disease progresses to a severe stage, then interventions and therapeutic approaches may not be very useful due to the unique nature of the structural properties of the synovial cavity articular cartilage. Several invasive and noninvasive diagnostic methodologies for detection have been developed or in the process of development, but they cannot diagnose early OA reliably, economically, and simply. Well-known techniques such as Xray, ${ }^{15}$ computed tomography, ${ }^{16}$ magnetic resonance imaging (MRI), ${ }^{17}$ and arthroscopy and arthrography ${ }^{18}$ are used for the diagnosis of KJO. Ultrasonography is relatively safe, inexpensive, and less time-consuming. However, the ultrasound technique is exceedingly dependent on the operator and its inability to assess deeper articular structures due to acoustic shadowing. ${ }^{18}$ However, these techniques have limitations and generally fail to detect early onset KJO. There is a need for a new detection methodology for the early diagnosis of osteoarthritis, so patients with a high risk of osteoarthritis progression can be provided early treatment interventions.

Bera $^{19}$ and Naranjo-Hernánde ${ }^{20}$ suggested that biological cells, containing intracellular fluids (ICF), cell membranes with or without cell walls, are suspended in the extracellular fluids (ECF) and during excitation show a frequency-dependent behavior to an alternating electrical signal. Under alternating electrical excitation, biological cells and tissues produce a complex bioelectrical impedance or electrical bioimpedance. $^{21}$ The bioelectrical impedance plethysmograph (BIP) diagnostic measurement technique is a complete, noninvasive, safe, simple, compact, and cost-effective diagnostic technique that can provide a useful methodology and fits very well the economically weaker sections of the population. In the present study, we used BIA for the diagnosis of knee OA. It is an easy, non-invasive, and relatively inexpensive technique that can be performed in almost any subject because it is portable. We suggest that, along with KL grade distribution scale measurement, the BIP approach has a clear potential to complement the OA diagnostic chain and make radiographic knee OA grading more objective.

\section{Experimental Procedure}

\section{Study Design}

Description of BIP

Bioelectrical impedance measurements are based on Ohm's law: Current in a circuit is directly proportional to voltage and inversely proportional to the resistance in a DC circuit or impedance in an alternating current (AC) circuit. Two electrodes are used to apply AC into the body or body segment. The voltage signal from the surface of the body is measured in terms of impedance using the additional two electrodes. $^{22}$

\section{Electrode System}

1. In an albino rat study: $2-3 \mathrm{~mm}$ thick single silver wire electrodes

2. In a bull calf study: Braided silver mesh wire current electrode (width: $1.5 \mathrm{~cm}$ and thickness: $4 \mathrm{~mm}$ ) and reactant (recording) electrode (width: $8 \mathrm{~mm}$ and thickness: 2$4 \mathrm{~mm})$.

3. In a human study: The width of current electrodes (width: $12 \mathrm{~mm}$ and thickness: $2-3 \mathrm{~mm}$ ) and reactant/recording electrodes (width: $8 \mathrm{~mm}$ and thickness: $2-3 \mathrm{~mm}$ ). The width of current electrodes was kept more to provide enhanced depth penetration of the current in the synovial cavity. The distance between the current and reactant electrodes was $\sim 2 \mathrm{~cm}$.

To confirm that the variations, which we observed by BIP technique, were due to alterations in knee synovial cavity, two preliminary experiments on animals (albino rats and bull calves) and human volunteers' were performed and pilot study results validated our contention that the impedance of the knee joint varies in animal studies and records do reflect some components of the synovial fluid and the AC.

\section{Animal Studies}

i. Albino rat knee joint study: A detailed experimental study was performed on five male albino rats weighing between 150 and $200 \mathrm{~g}$. Animals were anesthetized and all muscles around the knee joint were removed. Silver wire electrodes were tied around the knee joint for the impedance was measured by BIP (specification: $4 \mathrm{~mA}$ and $50 \mathrm{kHz}$ ), and the basal value of the rat's knee joints was recorded and an impedance of 4-6 $\mathbf{\Omega}$ was obtained. Subsequently, a tuberculin syringe was inserted into the synovial cavity of the rat to ensure that the movements of the joint were restricted. The synovial fluid was then removed from the knee joint cavity, and normal saline solution was gently perfused into the knee joint cavity of rats. Again, the impedance values were monitored on the BIP. Impedance changes were again observed and found to be in the order of -10 ohms.

ii. Bull calve knee joint study: Freshly amputated knee joints of three bull calves (age: 1-2 y) were obtained from a local slaughterhouse. Bull calve experiments were performed in a neutral environment. Resistance to electrical resistance of the knee joints of bull calves was measured before and after stripping of the skin and muscle layers. Four braided band silver electrodes were tied after applying a silicone conductive gel around the knee joint capsule above and below the lower end of the femur and proximal end of the tibia. The current and impedance electrode distances were kept at a distance of $2 \mathrm{~cm}$, whereas the pickup electrodes were kept as close as possible to the knee joint capsule. The base value of electrical impedance was measured by BIP (specification: $4 \mathrm{~mA}$ and $50 \mathrm{kHz}$ ) to 
detect the current status in the synovial cavity of the knee joints before and after the stripping of the joint capsule. To measure the variations in the interior milieu properties, $5 \mathrm{~mL}$ of normal saline was injected using a $10 \mathrm{~mL}$ syringe by slowly injecting into the synovial cavity of the bull calves knee joint. After some time, the fluid inside the joint cavity was aspirated by inserting the same syringe. A marked variation in the impedance was observed in the bull knee joint before and after saline injection in the synovial cavity, that is from $534 \Omega$ to $210 \Omega$ in the repeated experiments. This indicates that saline causes an increase in the conductivity inside the synovial cavity and decreases the impedance of the joint.

Human volunteer pilot study: In five clinically normal male human volunteers, knee joints (age: 40-50 years) were selected for a human pilot study to measure electrical impedance using the BIP technique (-Fig. 1). A 30-minute adaptation and acclimatization period was provided to eliminate localized muscular effects and equilibrate ionic imbalances. Two thick cotton cuffs containing braided silver wire mesh band electrodes were mounted above and below the knee joint after proper cleaning with a rectified spirit of the knee joint capsule and its surrounding areas (lower extremity of femur and upper extremity of tibia). An electrocardiogram (ECG) gel was applied to the silver wire electrodes before wrapping around the leg. Normal Subjects showed regular minor fluctuations in the basal recording. To understand the reason behind these minor fluctuations, a pressure cuff was placed around the mid-thigh region and the blood supply to the knee joint was occluded. The recording was again performed during occlusion, still, minute changes were observed before and after occlusion in basal values, suggesting that basal resistive values of electrical impedance will not be affected by minor pulsatile blood flow pulses and will not be able to modify the loading variations.

The results of the above-mentioned animal and human pilot studies confirm that the BIP can be extended for the assessment of osteoarthritic subjects. Hence, the BIP tech-

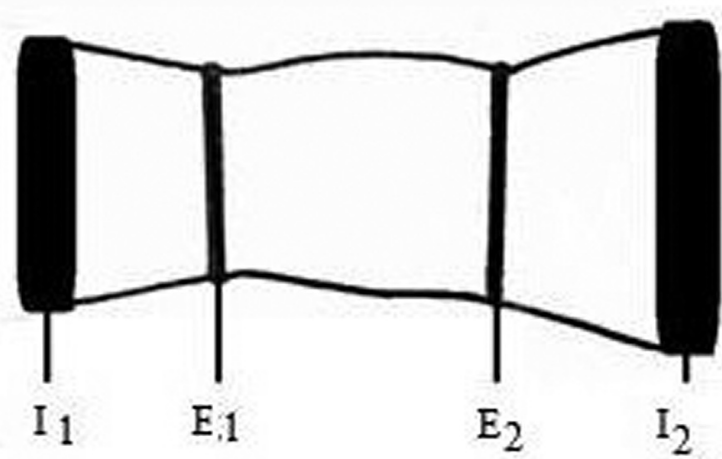

Fig. 2 Position of electrodes in the knee joint capsule.

nique has been used to measure the intrinsic properties of the synovial cavity of osteoarthritic human subjects.

Configuration of braided silver wire band electrode in human subjects: width of current electrodes (width: $12 \mathrm{~mm}$ and thickness $2-3 \mathrm{~mm}$ ) and reactant/recording electrodes (width: $8 \mathrm{~mm}$ and thickness $2-3 \mathrm{~mm}$ ). The width of the current electrodes was maintained to enhance the depth penetration of the current in the synovial cavity. The distance between the current and reactant electrodes was $\sim 2 \mathrm{~cm}$ (-Fig. 2).

\section{Clinical Study of Normal and Osteoarthritic Human Subjects}

Clinical investigation on osteoarthritic subjects, 15 healthy and 15 clinically diagnosed osteoarthritic volunteer knee joints were studied by impedance plethysmography. Anthropometric measurements of the legs were performed to predict the approximate impedance of the knee joint using computer analyses.

Human subjects (normal subjects age: $45 \pm 5.78$ and knee joint OA subjects $57.5 \pm 2.34 \mathrm{y}$ ) leg volume constituents were skin, muscle, bones, and blood. Bodyweight (normal [55 \pm 7.83 ], KJO subjects [68 \pm 9.52$])$.

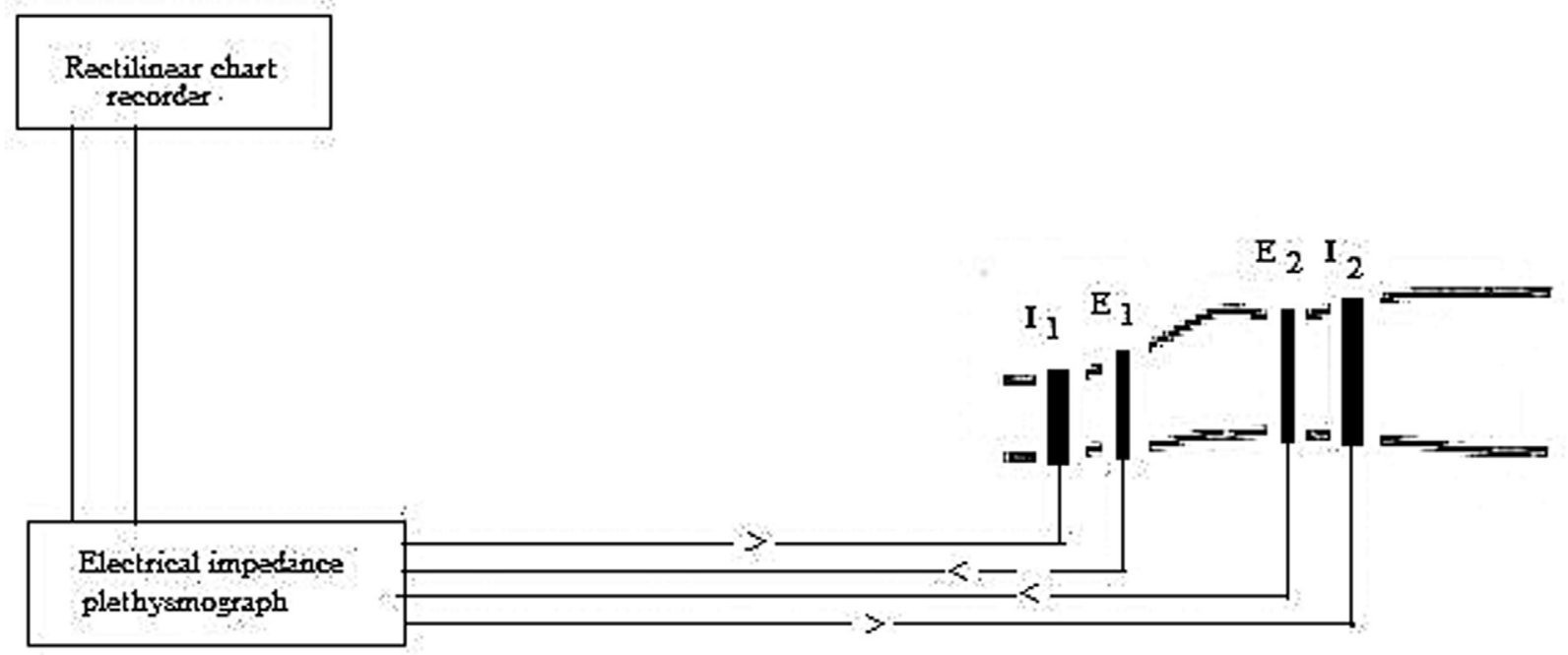

Fig. 1 Schematic representation of setup for knee joint. 


\section{Subjects Selection}

1. Normal control group (NCG): 15 male normal volunteers (age: 40-50 y) before induction were clinically assessed for no symptoms of knee joint disease on radiography or any other ailment of the knee joint.

2. Knee joint osteoarthritic group (KOG): 15 male patient volunteers (age: 50-65 y) Clinically diagnosed (symptomatologically and radiographically) was selected for the knee joint OA study. OA patients were obtained from different hospitals in Delhi (India).

\section{Experimental Protocol of Clinical Subjects}

Subjects of both groups (NCG and KOG) were asked to relax for 20 to 30 minutes to eliminate localized muscular effects and equilibrate ionic imbalances. To avoid any motion artifacts, all subjects in the NCG and KOG groups were asked to sit on a $2.5 \mathrm{~m}$ long, width $1.5 \mathrm{~m}$, and height $1.5 \mathrm{~m}$ wooden table with their legs stretched longitudinally on the table. A wooden plank (length: $2 \mathrm{~m}$ width, $1.5 \mathrm{~m}$ length) fixed on the back of the wall was used to support the hips and spinal region of the subjects with the knee fully flexed on the wooden table $\left(90^{\circ}\right.$ angle) to provide support to the back. Thick fabric cotton fixed E1 and I1 and E2 and I2 electrodes after applying sufficient electrode jelly were wrapped around the knee joint on the surface of skin joint capsule upper and lower ends of the proximal tibia and lower extremities of the femur, as close as possible. Velcro was used to tie the electrodes around the knee region. Impedance variations due to two loadings were measured by a $4 \mathrm{~mA}$ constant current source of $50 \mathrm{kHz}$ by BIP for 5 minutes. After the initial basal recording of electrical signals, loading regimen (compressive and tractive loading) experiments were performed under the supervision of trained technicians.

\section{Static and Dynamic Loadings in NCG and KOG in BIP}

Two types of loadings protocols were used for NCG and KOG subjects in the experimental protocol for assessing and recording the changes in the synovial joint capsule. Changes in the impedance were recorded during the application of the load and relaxation phases.

(a) Traction loading: Extension of the knee joint by applying a load of $10 \mathrm{~kg}$ on the ankle: Pulling (tractionloading) was applied by a halter tied and fixed around the lower tibial extremity and ankle via a cord running over a low friction pulley and $10 \mathrm{~kg}$ of the load was applied, and the responses of traction forces were recorded after 10 minutes (stabilization time; -Fig. 3 ).

(b) Compression loading: Pushing (compressive) loading was applied through a $10 \times 4$ inch flat wooden plank pressed against the sole of the foot using an electric jack (Pilot Q-HY-1500L 12 V, USA). The force is transmitted along the longitudinal axis of the leg. A compressive force of varying magnitude from $10 \mathrm{~kg}$ was applied and released after 2 minutes and monitored and recorded (-Fig. 4).

To ascertain that variations were due to tissue pathophysiology, anthropometric measurements of the two legs were taken to predict the approximate impedance of the knee joint

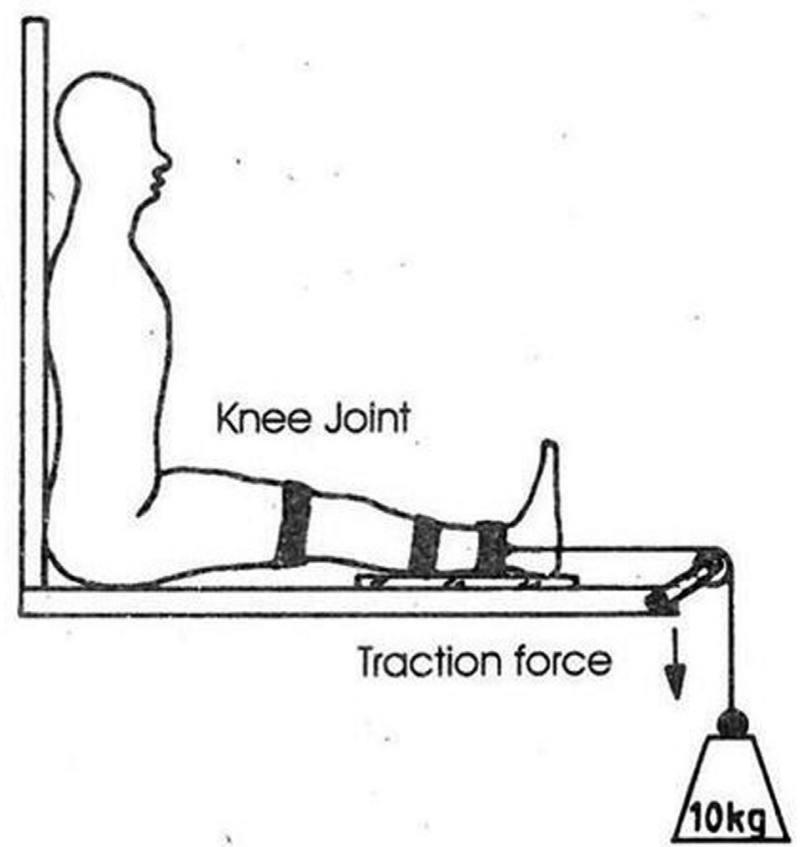

Fig. 3 Subjects position with traction halter knee Joint ( $10 \mathrm{~kg}$ load).

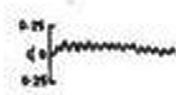

Normal knee joint

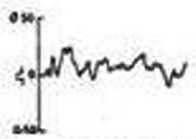

Osteoarthritic Knee joini

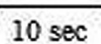

:
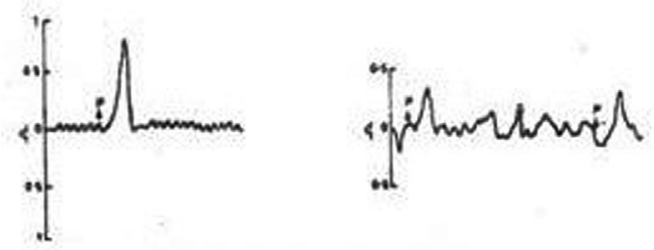

Traction Loading $(10 \mathrm{~kg})$
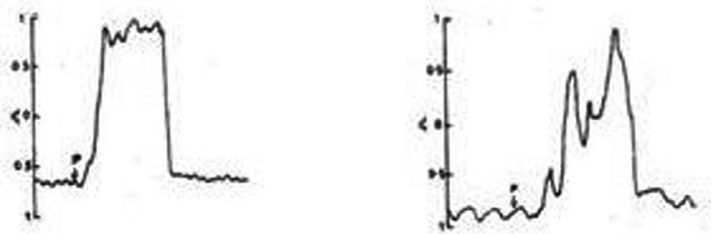

Compression Loading (10 kg)

Fig. 4 Dynamic load and resting potential response of normal and osteoarthritis knee joints.

by computer analysis. In addition, in every regimen phase, recordings were performed until a consistent waveform pattern of 1 minute was obtained. The subjects' rectilinear graphical recordings were analyzed by directly measuring 
the area bounded by an irregular curve using a mathematical polar planimeter instrument (Crosby Steam Gage \& Valve Co.. Boston, USA). Planimeter only helps in the measurement of surface area on a recording sheet to calculate the area for the assessment of changes in normal and OA subjects. Only the resistive component of the impedance by the BIP was measured and utilized for the assessment of the status of the interior milieu of the knee joint synovial cavity. Basal values were obtained by dividing the value of the balance point value to eliminate subject bias due to complicated contours and knee dimensions during normalized resistance fluctuations. Variations in waveform amplitude change and the area under the curve following loading and recovery states were calculated using a planimeter. In addition, the area of deviations in waveform recording on the rectilinear recorder below the baseline as well as above the baseline was considered positive and added to the cumulative total. For standardization, the fluctuations that fall within a time domain, four times the time duration of the wave corresponding to the loading as determined from zero-crossing were included in the fluctuations. Thereafter, smaller waves were neglected. Balance point assessment of resistive components divided by mean basal values (quotient) provided the resistive value, which helps to eliminate subject bias due to complicated contours and knee dimensions. In our study, the highest waveform amplitudes obtained during the initial and loading recovery phases were measured using a planimeter. During planimeter measurement, the areas for deviations below the baseline and above the baseline were considered positive and added to the cumulative total. For standardization, fluctuations that fell four times during the waveform due to the loading from zero-crossing were included, and smaller waves were neglected.

\section{Statistical Analysis of Data}

Statistical analysis of data was performed using the Microsoft Excel Spreadsheet for mean, mode, median analysis, and paired Student's $t$-test to assess the significance obtained. Resistance (capacitance) and waves $\left(\mathrm{cm}^{2} / 15 \mathrm{sec}-\right.$ onds from planimeter obtained from the rectilinear recorder and bioelectrical impedance plethysmograph of knee joint used for the calculation. Healthy and OA subjects data were used for analysis standard Deviation and $p$-value. The clinically relevant difference data between basal (resting) and loading (tractive and compressive) was studied and recorded. Based on this assumption, independent Student's $t$-test, $5 \%$ level of and test strength of 0.90 , at given strength of population of 30 normal and OA subjects were calculated by analysis of variance (ANOVA). A paired $t$-test (basalloading) was used to analyze the outcome at 12 months.
A two-tailed $(\alpha=0.5)(p<0.05)$ was considered statistically significant.

\section{Results}

The study was done to measure electrical impedance variations of normal and OA subjects at resting (basal) and loading conditions by noninvasive BIP technique to confirm that bioelectrical impedance plethysmographic method can detect properties of internal synovial cavity material changes by surface electrodes for diagnosing OA. The results of the above-mentioned animal and human pilot studies confirmed that the bioelectrical impedance technique can be extended for the assessment of osteoarthritic subjects. Normal subjects showed regular minor fluctuations in the basal recording.

To confirm that the variations we observed by our BIP technique were from the knee joint synovial cavity, two preliminary experiments on animals (albino rats and bull calves) and human volunteers were performed. The pilot study results validated our contention that the impedance of the knee joint varies in animal studies and records do reflect some components of the synovial fluid and the AC. Hence, the BIP technique has been for the measurements of intrinsic properties of the synovial cavity of osteoarthritic human subjects.

Tetra-polar electrodes were used to measure electrical impedance plethysmography signals around the knees of subjects in the normal knee and KJO subjects by providing an input current of $4 \mathrm{~mA} 50 \mathrm{kHz}$ by the BIP instrument. Measurement and data recordings were assessed using two inner electrodes in the display system in the machine to show the values in ohms $(\Omega)$ and variations in the waveform in rectilinear recorder and calculated by a planimeter.

- Table 1 presents the resistive data and base value (resting recording) of all normal and showed an average of $219.37 \pm 12.48 \Omega$, whereas osteoarthritic subjects showed $278.68 \pm 18.67 \Omega$ measured after the stabilization of subjects in the laboratory environment, i.e., after 30 minutes for 5minute assessment. The compressive loading was $219.47 \pm 22.34$ to $994.63 \pm 218 \Omega$ when compared with OA subjects, i.e., $\sim 4$ times increased in comparison with normal healthy knee joint subjects and tractive loading showed a reactant value of $378.79+78.46 \Omega$ in normal subjects compared with $767.38+130.67 \Omega$ in KJO subjects.

- Table 2 shows the mean average area measurements results obtained by planimeter (surface area covered by waveform for 15 seconds in $\mathrm{cm}^{2}$ ) value of the knee joint in compressive loading of the leg $(10 \mathrm{Kg})$ after planimeter calculation showed amplitude area: $\mathrm{cm}^{2} / 15$ seconds;

Table 1 Mean variation of impedance responses ${ }^{*} \Omega+$ in compressive and tractive loadings of normal and osteoarthritis subjects

\begin{tabular}{|l|l|l|l|l|}
\hline S. No. & Loading regimen & $\begin{array}{l}\text { Healthy subjects } \\
{ }^{*} \Omega+\end{array}$ & $\begin{array}{l}\text { Osteoarthritic subjects } \\
{ }^{*} \Omega+\end{array}$ & $p$-Value \\
\hline 1 & In traction position $(10 \mathrm{~kg}$ load) & $378.79+78.46$ & $767.38+130.67$ & $<0.001$ \\
\hline 2 & In compression position $(10 \mathrm{~kg}$ load) & $219.47+22.34$ & $994.63+218.48$ & $<0.001$ \\
\hline
\end{tabular}


Table 2 Surface area measured by the planimeter ( $\mathrm{cm}^{2} / 15$ seconds) at basal level and during tractive and compressive loadings and recorded on the rectilinear recorder by BEP

\begin{tabular}{|l|l|l|l|l|}
\hline S. No. & Loading regiment protocol & $\begin{array}{l}\text { Normal knee joint } \\
(\boldsymbol{n}=\mathbf{3 0})\end{array}$ & $\begin{array}{l}\text { Osteoarthritic knee joint } \\
(\boldsymbol{n}=\mathbf{3 0})\end{array}$ & $\boldsymbol{p}$-Value \\
\hline 1 & Compressive loading of the leg $(10 \mathrm{Kg})$ & $34.14 \pm 09.19$ & $2010.56 \pm 46.26$ & $<0001$ \\
\hline 2 & Tractive loading of leg $(10 \mathrm{Kg})$ & $67.00 \pm 25.26$ & $164.67 \pm 34.61$ & $<0.001$ \\
\hline
\end{tabular}

$34.14 \pm 09.19$ and $201.56 \pm 46.26$ in the KJO subjects. The tractive loading of the leg $(10 \mathrm{Kg})$ demonstrated a mean area value of $164.67 \pm 34.61$ in comparison to normal subjects' assessment value of $67.00 \pm 25.26$ in $\mathrm{cm}^{2}$ for 15 seconds.

\section{Discussion}

The objective of this study was to develop a non-invasive, simple, and cost-effective diagnostic methodology for slowing the progress of disabling joint disorder, so active movable life continues for a long time. Several diagnostic techniques have been developed to diagnose the loadbearing synovial joint properties (radiography, arthrography, MRI, ultrasound sonography, and optical coherence tomography). These detection methodologies have several limitations and cannot provide an on-time reliable diagnosis. Due to the complexity of joint disorders and their prevalence, several studies have been conducted to understand their genesis, etiology, and physiopathology as well as its diagnosis and remedial therapies. Du et al found that almost a quarter of patients with $\mathrm{OA}$ on radiographic images do not complain about the symptoms of the disability. ${ }^{23}$ Because of several discrepancies in the diagnosis of KJO, researchers are now focusing more on prevention and treatment in the early stages of the disease. ${ }^{24}$

Early detection of $\mathrm{OA}$ is necessary because a literature survey showed that advanced degradation of hyaline $A C$ is untreatable. Load-bearing AC is a dynamically active avascular aneural tissue and depends on the surrounding materials (synovial fluid). If the OA disease process is detected at early stages, then the progress of degradation can be prolonged, giving more years of a good life. Several conventional and nonconventional diagnostic techniques such as arthrography, MRI, ultrasound sonography, and optical coherence tomography (OCT) are available. It is found that present diagnostic methodologies are not able to detect OA perfectly and have several shortcomings and cannot be used and efficiently further limit their use in some way. The present diagnostic tools are not compact (movement difficulty) or expensive. In addition, they cannot detect $\mathrm{OA}$ at the early stages of development. OA is most prevalent in developing and poor countries in Asia and Africa, where economic conditions and health services are in poor shape. A literature survey revealed that the BIP technique can be used to understand load-bearing joints. AC degeneration and degradation can be simply, safely and efficiently measured by bioelectrical impedance plethysmography and has received immense support for studying movable joint pathophysiology. ${ }^{25-28}$
Therefore, the present study was conducted to measure electrical impedance variations of normal and OA subjects at resting (basal) and loading conditions by noninvasive BIP technique for the early onset of $\mathrm{OA}$. To confirm that the bioelectrical impedance plethysmographic method was used to measure the internal properties of the synovial cavity by surface electrodes for diagnosing $\mathrm{OA}$, we performed two important preliminary experiments on animals (albino rats and bull calves) and human volunteers. Pilot studies indicated that the impedance of the knee joint varies in animal studies and records do reflect some components of the synovial fluid, and the ACs and BIP technique can be used for the measurement of intrinsic properties of the synovial cavity of humans.

In our normal volunteers, the pilot study showed minute cardiac pulsatile blood flow oscillations in waveform recording but minor pulsatile fluctuations were not altered during cartilaginous stresses/loading measurements. In addition, a human control study showed similar reactant values before and after occlusion. Hence, as the biophysical assumptions postulated earlier, a human pilot study confirmed the efficacy of measurement by BIP of the knee joint and due to alterations in the environment of the synovial cavity (articular cartilage/synovial fluid) rather than by other tissue structures and intervening variables. Resistance measurement and frequency waveform recording showed significant differences between normal and osteoarthritic subjects. Resistance assessment showed $p$-value of $<0.001$ (-Table 1, -Fig. 5). Rectilinear chart recorder waveform

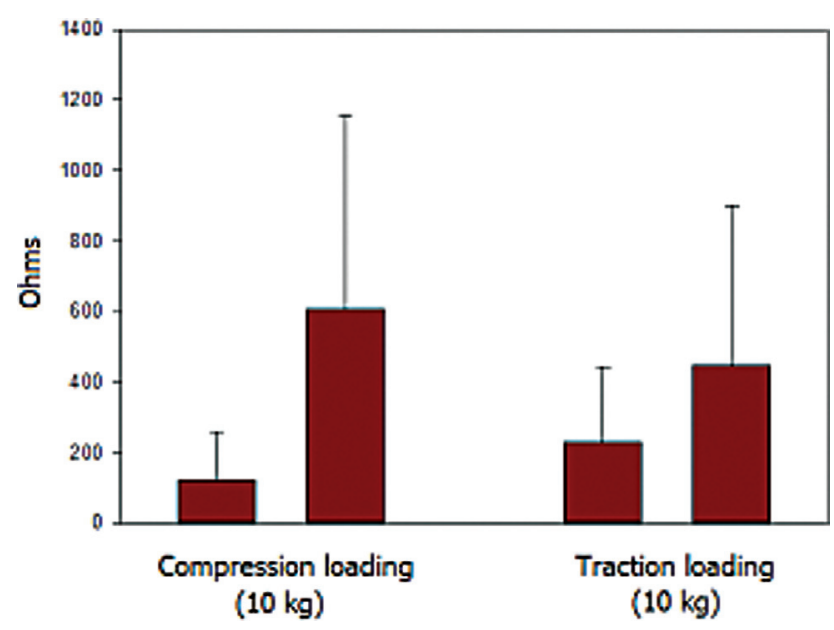

Fig. 5 Bar graphs showing mean values of resistance obtained on display board (ohms). 


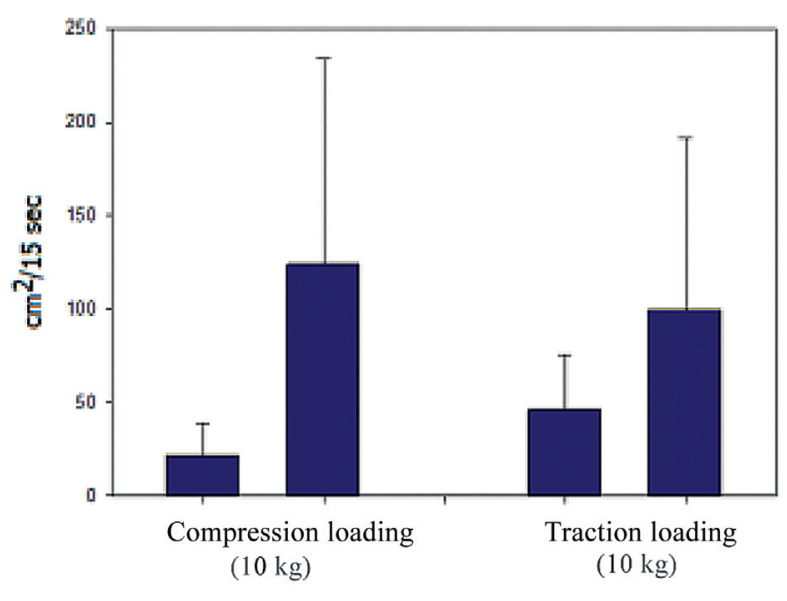

Fig. 6 Bar graphs showing mean values of waveform recording on rectilinear recorder measured by Planimeter ( $\mathrm{cm}^{2} / 15$ seconds) at basal level and during tractive and compressive loadings and recorded on the rectilinear recorder by bioelectrical plethysmograph.

analysis showed $p<0.001$ in compression and traction loading regimen (-Fig. 6).

Loadings of joints provide significant variations; during compressive loading, stimulation produced more variations than tractive loading regimens situation. This variation may be due to different flow properties of the fluid in intrinsic contents of the synovial cavity. A significant value of $(p<0.001)$ was observed in both regimens.

KJO subjects showed an $\sim 60 \%$ increase in the waveform during the compressive phase of loading compared with the normal subject's knee joint. However, tractive loading showed an $\sim 30 \%$ increase in OA in the waveform frequency to stress change compared with normal subjects. Percentage comparison of compressive and tractive loadings showed marked differences in both groups of subjects. The difference in the waveform observed in BIP recording in compressive and tractive loading may be due to different environments developed in stress conditions and the flow of conducting fluids in and out of the corroded channels of the articulating surfaces. The osteoarthritic group of subjects showed large and high-wave amplitude (oscillation) during loading in the stress phase, it does reflect a resistive waveform pattern compared with normal subjects.

The results sometimes observed intersection of basal values of the electrical impedance between healthy control and osteoarthritic subjects, suggesting that basal impedance reflects the bulk resistance component of the tissue was examined and it is governed by the anthropometric dimensions of the structure. In addition, in our study, only the resistive component of the impedance was utilized for the assessment, because resistive alterations provide the actual effect of loading regimen on the knee joint, and it helps to eliminate subject bias on account of complicated contours and knee dimensions. All waveform amplitude frequencies obtained during initial, loadings, and recovery phases were measured by a planimeter, and the areas for deviations below the baseline and above the baseline were considered positive and added to the cumulative total. For standardization of values, fluctuations that fall four times during the waveform due to the loading from zero-crossing were included, and smaller waves were neglected. However, numerous bioimpedance studies have been performed to evaluate the internal physiopathology of the body noninvasively using surface electrodes. However, a literature survey showed that there are very few studies have assessed the OA noninvasively by BIP.

Distefano et al studied the effect of pneumatic tourniquet on limb circulation during ischemia and swelling by electrical impedance plethysmography (EIP) and found that EIP is an accurate, noninvasive, and simple method to record the increasing periods of a tourniquet and evaluate preventative measures as well as treatment. ${ }^{29}$ Alvarenga et al compared human OA with normal healthy knee subjects and found a significant difference between them $(p<0.01)$ and suggested bioelectrical impedance can be used to diagnose and determine the inflammatory pathological conditions of the knee joint. ${ }^{30}$ Later on, Neves et al studied $\mathrm{OA}$ and showed that bioimpedance is a sensitive technique to assess the physiological changes associated with OA and suggested that the BIS technique can provide a noninvasive evaluation of the diagnosis of knee OA. ${ }^{31}$ In our study, the main emphasis was to understand that whether BIP methodology could measure the impedance in the knee joint. In addition, a major question was whether the variations were due to changes within the synovial cavity or due to configurational changes in the muscle and other structures in the joint. These structures can affect the impedance changes during the recording of the changes in the synovial cavity, but during the loading regimen, the intervening variables changes obtained were in the articular region. Animal studies of rat and bull knee joints validated our view. These results indicated that the changes observed in our BIP study for the electrical impedance assessment of the synovial cavity were due to the flow of synovial fluid mobile ions into the porous charged matrix of the $A C$, which produced a change in the conductance due to the flow of the fluid. ${ }^{32-35}$ Our study recorded oscillatory type of response in all arthritic patients during the entire phase of recording, but normal healthy joint subjects showed only low-amplitude pulsatile blood flow during the resting phase of recording. We believe that oscillations in $\mathrm{OA}$ subjects may be due to transport delays in intra- and extracellular fluid mobilization in the articular membrane. Earlier studies also showed that corrosion and degradation of $\mathrm{AC}$ interrupt the free flow of synovial fluid ions in $\mathrm{OA}$ cartilage, causing the change in the conductivity of the region. ${ }^{28,36}$ Assessment procedure done by us using electrical impedance detection diagnostic technique can display its scientific efficacy, and sensitivity in comparison to other sophisticated diagnostic technologies is presented. It is suggested that BIP can be used along with other diagnostic technologies for the detection and diagnosis of various ailments. 


\section{Conclusions}

The results of the present study indicate that the BIP methodology of diagnosis can measure impedance variations. This technique can be used to reflect the degenerative conditions of the knee joints before the detection of the disease by other clinical methodologies and the analysis of data suggests that extracellular AC matrix resistance and reactance components can evaluate the intensity of KJO. Overall, the BIP detection technique may provide an alternative noninvasive method for the diagnosis of KJO. BIP is a simple, safe, and cost-effective diagnostic method. The results of this study and experimental data confirm that the proposed method of bioelectrical impedance evaluation, when used for the diagnosis of the knee, is a valid and reliable method to determine the pathological conditions of the knee joint. In addition, the cost-effectiveness of BIP technology may help reduce the health burden of knee OA. The articular cartilage of the joint is a dynamic tissue that responds to changes in loading conditions. It is well accepted that regular loading of AC within physiological limits throughout life is necessary to maintain normal joint homeostasis. The bioelectrical impedance of the knee was assessed using tetrapolar silver band electrodes. A constant current of $3 \mathrm{~mA}$ at $20 \mathrm{kHz}$ was generated by BIP. Impedance was measured by balancing the resistive and responsive components on a rectilinear recorder. Normal and clinically diagnosed KJO subjects were studied in compressive and traction phases and showed significant differences in the properties of electrical signals.

\section{Conflict of Interest}

None declared.

\section{References}

1 Mukhopadhaya B, Barooah B. Osteoarthritis of the hip in Indians: an anatomical and clinical study. Indian J Orthop 1967;1:55-63

2 Gunn DR. The comparative rarity of primary degenerative osteoarthritis of the hip joint in south and East Asia and a possible explanation of this observation. Indian J Orthop 1969;3:76-88

3 Srikanth VK, Fryer JL, Zhai G, Winzenberg TM, Hosmer D, Jones G. A meta-analysis of sex differences prevalence, incidence and severity of osteoarthritis. Osteoarthritis Cartilage 2005;13(09): 769-781

4 Zhang Y, Jordan JM. Epidemiology of osteoarthritis. Clin Geriatr Med 2010;26(03):355-369

5 Conley S, Rosenberg A, Crowninshield R. The female knee: anatomic variations. J Am Acad Orthop Surg 2007;15(Suppl 01):S31-S36

6 Wluka AE, Cicuttini FM, Spector TD. Menopause, oestrogens and arthritis. Maturitas 2000;35(03):183-199

7 Sophia Fox AJ, Bedi A, Rodeo SA. The basic science of articular cartilage: structure, composition, and function. Sports Health 2009;1(06):461-468

8 Torzilli PA. Influence of cartilage conformation on its equilibrium water partition. J Orthop Res 1985;3(04):473-483

9 Lai WM, Hou JS, Mow VC. A triphasic theory for the swelling and deformation behaviors of articular cartilage. J Biomech Eng 1991; 113(03):245-258

10 Mow VC, Holmes MH, Lai WM. Fluid transport and mechanical properties of articular cartilage: a review. J Biomech 1984;17(05): 377-394
11 Frank E, Evans R, Lee C, Treppo S, Spector M, Grodzinsky A. Quantitative electrical impedance analysis of cartilage degradation. Biorheology 2004;41(3-4):195-202

12 Saxena RK, Sahay KB, Guha SK. Morphological changes in the bovine articular cartilage subjected to moderate and high loadings. Acta Anat (Basel) 1991;142(02):152-157

13 Taylor AM, Boyde A, Davidson JS, Jarvis JC, Ranganath LR, Gallagher JA. Identification of trabecular excrescences, novel microanatomical structures, present in bone in osteoarthropathies. Eur Cell Mater 2012;23:300-308, discussion 308-309

14 Fernandes JC, Martel-Pelletier J, Pelletier JP. The role of cytokines in osteoarthritis pathophysiology. Biorheology 2002;39(12):237-246

15 Bedson J, Croft PR. The discordance between clinical and radiographic knee osteoarthritis: a systematic search and summary of the literature. BMC Musculoskelet Disord 2008;9:116

16 Jahr H, Brill N, Nebelung S. Detecting early stage osteoarthritis by optical coherence tomography? Biomarkers 2015;20(08): 590-596

17 Alizai H, Roemer FW, Hayashi D, Crema MD, Felson DT, Guermazi A. An update on risk factors for cartilage loss in knee osteoarthritis assessed using MRI-based semiquantitative grading methods. Eur Radiol 2015;25(03):883-893

18 Hugo PC III, Newberg AH, Newman JS, Wetzner SM. Complications of Arthrography. Semin Musculoskelet Radiol 1998;2(04): 345-348

19 Palmer AJ, Brown CP, McNally EG, et al. Non-invasive imaging of cartilage in early osteoarthritis. Bone Joint J 2013;95-B(06): 738-746

20 Bera TK. Bioelectrical impedance methods for noninvasive health monitoring: A review. J Med Eng 2014;2014:381251

21 Naranjo-Hernández D, Reina-Tosina J, Mart MinM. Fundamentals, recent advances, and future challenges in bioimpedance devices for healthcare applications. Hindawi J Sensors 2019;9210258: $1-42$

22 Khan M, O'hara R, Pohlman RL, et al. Multi-dimension applications of bioelectrical impedance analysis. Journal of Exercise Physiology 2005;8:56-71(JEP online)

$23 \mathrm{Du} \mathrm{H}$, Chen SL, Bao CD, et al. Prevalence and risk factors of knee osteoarthritis in Huang-Pu District, Shanghai, China. Rheumatol Int 2005;25(08):585-590

24 Glyn-Jones S, Palmer AJ, Agricola R, et al. Osteoarthritis. Lancet 2015;386(9991):376-387

25 Ackmann JJ, Seitz MA. Methods of complex impedance measurements in biologic tissue. Crit Rev Biomed Eng 1984;11(04): 281-311

26 Kushner RF. Bioelectrical impedance analysis: a review of principles and applications. J Am Coll Nutr 1992;11(02):199-209

27 Kyle UG, Bosaeus I, De Lorenzo AD, et al; ESPEN. Bioelectrical impedance analysis-part II: utilization in clinical practice. Clin Nutr 2004;23(06):1430-1453

28 Vasold KL, Parks AC, Phelan DML, Pontifex MB, Pivarnik JM. Reliability and validity of commercially available low-cost bioelectrical impedance analysis. Int J Sport Nutr Exerc Metab 2019; 29(04):406-410

29 DiStefano V, Nixon JE, Stone RH. Bioelectrical impedance plethysmography as an investigative tool in orthopaedic surgery. A comparative study of limb exsanguination techniques. Clin Orthop Relat Res 1974;(99):203-206

30 Alvarenga RL, Souza MN. Assessment of knee osteoarthritis by bioelectrical impedance. In: Proceedings of the 25th Annual International Conference of the IEEE Engineering in Medicine and Biology Society. ; 2003 Sept 17-21; Cancun, Mexico: IEEE; 2003:3118-3121

31 Neves EB, Pino AV, de Almeida RM, de Souza MN. Knee bioelectric impedance assessment in healthy/with osteoarthritis subjects. Physiol Meas 2010;31(02):207-219 
32 Lee RC, Frank EH, Grodzinsky AJ, Roylanc DK. Oscillatory compressional behavior of articular cartilage and its associated electromechanical properties. Transactions of the ASME 1981;103:280-292

33 Levick JR, McDonald JN. Fluid movement across synovium in healthy joints: role of synovial fluid macromolecules. Ann Rheum Dis 1995;54(05):417-423

34 Minassian A, O’Hare D, Parker KH, Urban JP, Warensjo K, Winlove $\mathrm{CP}$. Measurement of the charge properties of articular cartilage by an electrokinetic method. J Orthop Res 1998;16(06):720-725
35 Saarakkala S, Julkunen P, Kiviranta P, Mäkitalo J, Jurvelin JS, Korhonen RK. Depth-wise progression of osteoarthritis in human articular cartilage: investigation of composition, structure and biomechanics. Osteoarthritis Cartilage 2010;18(01): 73-81

36 Hui AY, McCarty WJ, Masuda K, Firestein GS, Sah RL. A systems biology approach to synovial joint lubrication in health, injury, and disease. Wiley Interdiscip Rev Syst Biol Med 2012;4(01): $15-37$ 